\title{
La monosémie des termes de l'anglais du droit, ou le raisonnement en termes de gradient de potentiel
}

The monosemy of English legal terms, or the recognition for a gradient potential in sense boundaries

\section{Andreas Pichler}

\section{OpenEdition}

\section{Journals}

Édition électronique

URL : http://journals.openedition.org/asp/5064

DOI : 10.4000/asp.5064

ISSN : 2108-6354

Éditeur

Groupe d'étude et de recherche en anglais de spécialité

Édition imprimée

Date de publication : 1 novembre 2017

Pagination : 49-67

ISSN : 1246-8185

Référence électronique

Andreas Pichler, «La monosémie des termes de l'anglais du droit, ou le raisonnement en termes de gradient de potentiel », ASp [En ligne], 72 | 2017, mis en ligne le 01 novembre 2018, consulté le 02 novembre 2020. URL : http://journals.openedition.org/asp/5064; DOI : https://doi.org/10.4000/asp. 5064

Ce document a été généré automatiquement le 2 novembre 2020.

Tous droits réservés 


\section{La monosémie des termes de l'anglais du droit, ou le raisonnement en termes de gradient de potentiel}

The monosemy of English legal terms, or the recognition for a gradient potential in sense boundaries

Andreas Pichler

\section{Introduction}

1 Cette étude vise à analyser la restriction de sens des termes appartenant au domaine du droit anglais ${ }^{1}$, plus précisément la procédure du conveyancing. Depuis la période féodale du moyen âge, cette procédure prend appui sur des termes spécialisés qui semblent tendre vers une dimension monosémique. La monosémie, caractéristique d'un signe ${ }^{2}$ qui ne possède qu'un seul sens, est un phénomène assez rare, y compris en anglais du droit, qui suppose l'hypothèse d'un raisonnement en termes « de gradient de potentiel », c'est-à-dire de taux de variation quant au potentiel monosémique. Le degré de spécificité d'un terme semble en effet subir des taux de variation d'une grandeur relativement importante en fonction de différents paramètres qui apporteraient chacun une certaine restriction de sens. La restriction de sens apparaîtrait donc comme un phénomène complexe, notamment en ce qui concerne les termes du conveyancing. Notre étude a pour but de faire émerger leur potentiel monosémique, potentiel qui se veut aussi une porte d'entrée vers le sens spécialisé du vocabulaire juridique.

2 Le corpus qui a nourri cette recherche se compose essentiellement de contrats du conveyancing qui dans la pratique sont caractérisés par une norme formelle unique. La forme standardisée fait de ces contrats des documents normés dont les termes désignent les conditions générales et spécifiques (standard form contract terms). Dans le 
cadre de ce travail, nous avons sélectionné une quarantaine de termes provenant d'environ 500 contrats publiés entre 2004 et 2016 par The Law Society (SCSLS) et les solicitors anglais $(\mathrm{CSRFC})^{3}$, contrats dont nous étudions les aspects sémantiques et pragmatiques depuis une dizaine d'années en anglais de spécialité et en tant que professionnel du domaine. Notre choix du domaine d'application du conveyancing est motivé par la portée linguistique, terminologique et pratique de termes que nous avons rencontrés lors de notre travail dans des études notariales en Angleterre et en France.

Les recherches contemporaines en anglais de spécialité ne paraissent que rarement s'intéresser au potentiel monosémique des termes juridiques. De nombreux travaux analysent essentiellement la sémantique à travers le phénomène de la polysémie des termes spécialisés (Ducrot \& Schaeffer 1972 ; Delavigne \& Bouveret 2000 ; Ferrari 2002 ; Condamines 2005 ; Cruse 2004). Dans de multiples études, la monosémie est un fait acquis au sein du vocabulaire scientifique (Baylon \& Mignot 2000 ; Depecker 2002), ce qui la range tout de même dans «le lot des langues spécialisées, non des langues en général " (Lerat 1997: 6). La monosémie a néanmoins été remise en question, en particulier par les diverses théories communicatives de la terminologie (Cabré 1998 ; Temmerman 2000). En France, la monosémie des termes spécialisés ne semble pas avoir encore reçu l'attention qu'elle mérite. La plupart des recherches terminologiques françaises sont de nature lexicographique, traductologique et contextuelle (Gledhill \& Kübler 2016 : 85-86). Certes, le conveyancing constitue en lui-même un domaine, qui de surcroît est particulièrement technique et aux contours très précis. En prenant compte de ce paramètre susceptible de favoriser la tendance à la monosémie, nous nous appuyons sur l'ensemble des paramètres linguistiques et terminologiques afin d'étudier le potentiel monosémique des termes appartenant à ce domaine du droit anglais.

Dans le droit civil de la common law, le nom conveyancing dénote la procédure translative de propriété par laquelle le titre d'acquisition d'un bien immobilier est transféré au nouveau propriétaire, soit par un acte de vente, une donation, une cession de parts sociales ou dans le cadre d'une succession. Cette procédure se divise en deux phases : d'abord, la formation du rapport contractuel repose sur les conditions de fond de formation et sur celles de forme qui peuvent être nécessaires à la formation valable de droit des obligations. Ainsi, les principes généraux de l'offre, de l'acceptation, de la capacité de contracter et de l'objet de l'obligation forment la matière de l'engagement. Ensuite, les effets de ce rapport contractuel donnent lieu à l'étude des effets du contrat entre les parties et à l'égard des tiers. L'acte juridique entame effectivement la force obligatoire du contrat qui, selon l'article 2 de la Law of Property Act (1929), dispose que «les conventions doivent être exécutées »4. À condition qu'il soit légalement formé, le contrat constitue ainsi une véritable loi régissant les contractants qui se caractérise par son format unique et son cadre formel très particulier s'agissant d'une publication par acte authentique auprès des instances compétentes. Dans le cadre limité de cette étude, nous avons fait appel à une sélection des termes du conveyancing appartenant seulement à la juridiction anglaise.

5 Après un bref rappel des questions théoriques, nous nous interrogeons d'abord sur les différentes sources du potentiel monosémique des termes du conveyancing, notamment pour tenter de déterminer si le recours à la racine morphologique peut être un argument en faveur d'une certaine restriction de sens. Ensuite, nous abordons les propriétés qualitatives des termes, et plus précisément celles de la référence et de la notion conceptuelle comme déterminants qualitatifs à la fragmentation du sens. Enfin, 
nous tentons d'éclaircir le rôle de la communication spécialisée en tant que critère décisif d'une possible monosémie. Cette démarche met, en outre, l'accent sur la différence entre une monosémie théorique et l'emploi monosémique des termes. Cependant, avant de répondre à ces interrogations, nous rappelons quelques fondamentaux théoriques.

\section{Cadre théorique : la monosémie entre linguistique et terminologie}

6 Le terme « sens » mérite d'être mis en question avant tout, car il appartient à la science des signes. En linguistique, et plus exactement en sémiotique, l'étude des signes concerne, depuis les propos de Ferdinand de Saussure dans son Cours de linguistique générale, cette entité double composée de deux éléments solidaires que sont la forme et la signification (Baylon \& Mignot 2000 : 17). La sémiotique a depuis adopté les termes saussuriens «signifiant » pour la forme et «signifié » pour la signification (Saussure 1995 [1916] : 99-100). Le sens fonctionne au sein de cette unité relationnelle dès lors que le signifié accorde au signifiant une valeur sémantique. Dans la recherche sémiologique contemporaine, le signe est le triplet formé du signifié, du signifiant et de ses règles d'emploi, car «tout mot a un sens, mais ce sens est une réalité psychologique » (Baylon \& Mignot op.cit.: 29). Au-delà des considérations interlinguistiques, la signification du signe s'appuie sur la réalité extralinguistique, d'où l'intérêt d'une relation triangulaire entre forme, sens et référent (Baylon \& Mignot op.cit. : 30). Le triangle sémiotique est un schéma qui prend en compte les réalités extralinguistiques par le biais du référent dépassant les simples frontières du système langagier.

7 Le renvoi à des réalités extralinguistiques est aussi un moyen d'illustrer les liens existants entre linguistique et terminologie de la même manière que le référent représente un élément clé en recherche terminologique. La référence à une réalité extérieure au système langagier situe la signification du signe dans le monde réel, et comme la terminologie a pour objet l'étude de la dénomination terminologique des concepts en rapport avec les domaines de savoir du monde réel, elle se fie davantage aux référents plus ou moins concrets. L'attention portée à l'égard du référent fait de la terminologie une science interdisciplinaire dans la mesure où elle intègre des aspects empiriques de la logique, des mathématiques, de la psychologie et de la cognition. Ces aspects servent sans doute à mieux définir les concepts spécialisés, mais avant tout, ils permettent de distinguer le sens des termes du sens général des mots de la langue courante. L'un des créateurs de la discipline, Eugen Wüster, fait d'ailleurs allusion à cette démarcation: "La langue se compose essentiellement de mots qui en tant que dénominations sont directement attribués aux concepts " $(1979: 6)^{5}$. La démarcation entre mot et terme est une frontière nécessaire dans notre étude sur le potentiel monosémique des termes du conveyancing car le signe considéré en lui-même présente des limites. Le terme constitue justement cette unité terminologique entre dénomination et concept qui ancre tout sens dans la réalité concrète d'un domaine professionnel. Par conséquent, nous utilisons le terme signification pour désigner le signifié d'un mot spécialisé, concept pour parler des notions de la réalité extralinguistique liées au mot, et référence afin de déterminer l'opération qui mène du concept au signe par le biais d'un référent. Ainsi, nous tenons compte de la réalité 
extralinguistique dans notre démonstration visant à mettre en évidence l'émergence progressive d'un potentiel monosémique.

Du grec monos «seul» et de semainein "signifier », la monosémie désigne, selon le Centre national de ressources textuelles et lexicales, un « caractère d'une unité significative, lexicale ou grammaticale, qui n'a qu'un seul sens $»^{6}$. Les autres unités, identifiées auparavant par Michel Bréal dans son Essai de sémantique, sont polysémiques et possèdent plusieurs sens ${ }^{7}$. Si la polysémie des mots est un phénomène largement répandu dans la langue générale, la monosémie y est plutôt rare (Cruse 2004 ; Depecker 2002 ; Lescot 2013). On la trouve en revanche dans les vocabulaires spécialisés, car "dans ces domaines, il est en effet nécessaire que les mots aient un sens précis " (Lescot 2013 : 121). Or, il est aussi parfois nécessaire qu'un terme n'ait pas de sens précis face aux besoins réels, même à l'intérieur du seul discours juridique du conveyancing. Par conséquent, la polysémie interne et externe est fréquente et la question se pose justement de savoir à partir de quel moment la polysémie cède la place à une monosémie potentielle. Quels sont les points de convergence et de divergence qui permettent de dessiner plus nettement les frontières théoriques entre l'aspect polysémique et la tendance à la monosémie d'un terme ? Dans la mesure où le sens de chaque terme est en étroite liaison avec le signe, il semble judicieux de considérer le potentiel monosémique, et plus précisément la restriction de sens, d'un point de vue à la fois linguistique et terminologique. Du fait que la monosémie est avant tout un phénomène linguistique, nous avons préféré commencer nos propos théoriques par une première interrogation sur les racines formelles des termes. À cet effet, les termes sont d'abord décontextualisés, puis recontextualisés dans le but de mieux illustrer la pertinence des divers paramètres linguistiques et terminologiques de la restriction de sens, mais aussi de placer la plasticité sémantique au cœur du débat.

\section{Le recours à la racine morphologique comme argument en faveur d'une restriction de sens dans les termes du conveyancing}

9 Pour toute analyse de sens des termes du conveyancing, il est nécessaire de commencer par la formation des unités significatives les plus petites, c'est-à-dire les entités morphologiques lexicales qui possèdent une signification. Le recours à la racine formelle de ces unités auquel Ducrot et Schaeffer consacrent un chapitre revêt le rôle du morphème "qui renvoie, à travers le grec, à l'idée de forme " (1972 : 432). Un morphème, souvent sous la forme d'un mot $^{8}$, peut être de catégorie grammaticale à l'exemple des terminaisons verbales et dans ce cas-là, il contribue à l'organisation grammaticale de la phrase. Ces mots-outils n'ont de sens qu'à l'intérieur du système langagier. Les morphèmes appartenant à la catégorie lexicale sont quant à eux porteurs de sens puisqu'ils constituent le lexique de la langue. Dans le cadre de notre étude, nous nous intéressons à ces morphèmes afin de déterminer si la morphologie peut être un argument en faveur d'une restriction de sens.

10 Les morphèmes que nous avons retenus comprennent une quarantaine d'entrées que nous avons préalablement organisées en fonction de leur typologique morphologique (Tableau 1). Les termes de la liste ci-dessous sont tous attestés par une définition dans Le grand dictionnaire terminologique (GDT) et dans Nolo's Free Dictionary of Law Terms and 
Legal Definitions (Nolo 2017). Toutes les informations terminologiques concernant les termes et leurs définitions en sont tirées. Nous avons relevé manuellement les termes afin de bien prendre en compte toutes les entrées essentielles à une éventuelle monosémie.

Tableau 1. Entrées prises en compte et classées en fonction de la typologie des morphèmes

\begin{tabular}{|l|l|}
\hline $\begin{array}{l}\text { Typologie des } \\
\text { morphèmes }\end{array}$ & Entrées prises en compte \\
\hline $\begin{array}{l}\text { Morphèmes } \\
\text { lexicaux libres }\end{array}$ & Title, contract, offer, term, conduct, clause, deed, search. \\
\hline $\begin{array}{l}\text { Morphèmes } \\
\text { composés, accolés } \\
\text { ou non accolés }\end{array}$ & $\begin{array}{l}\text { Mortgage, freehold, leasehold, title abstract, title number, title plan, title } \\
\text { report, title search, purchase title, real estate, real property. }\end{array}$ \\
\hline $\begin{array}{l}\text { Morphèmes } \\
\text { dérivationnels }\end{array}$ & $\begin{array}{l}\text { Realty, property, certainty, personality, capacity, consideration, completion, } \\
\text { execution, conveyance, conveyancing, agreement, acceptance, intention, } \\
\text { performance, incorporation, delivery, easement, encumbrance, seller, } \\
\text { buyer. }\end{array}$ \\
\hline
\end{tabular}

11 La liste du tableau 1 ne prétend en aucun cas à l'exhaustivité, mais elle permet toutefois de distinguer différents types de morphèmes, indépendamment du domaine de spécialité auquel ils se rapportent. Ces types concernent les morphèmes libres, dérivationnels, composés et ceux des syntagmes. ${ }^{9}$. Ceux qui servent d'exemple dans notre démonstration figurent en italique dans le tableau 1.

Tout d'abord, la forme minimale d'un mot peut concerner des mots simples, non décomposables comme deed ${ }^{10}$, et des mots tels que freehold, décomposables en unités significatives plus petites. Les deux exemples possèdent des significations plus ou moins spécifiques. Dans le domaine du droit des contrats, le mot deed fait généralement référence au contrat juridique alors que freehold désigne l'une des manières possibles d'acquérir un bien immobilier. Quant à leur formation, le mot freehold en particulier correspond à un morphème lié puisqu'il se compose de deux sémantèmes accolés directement l'un à l'autre qui se construisent à partir de deux bases non dérivées, free (N1) et hold (N2) dont N1 est un adjectif et N2 un nom déverbal au participe passé. Ces deux sémantèmes, parties du mot porteuses d'une signification lexicale, se combinent entre eux selon l'ordre déterminant-déterminé ; ils se composent d'un élément central, la partie tête, et d'une partie non-tête, l'adjectif free, qui spécifie une sorte de classe, ici le type de possession. Ce mot composé est endocentrique dans mesure où la partie tête, porteuse de sens principal, est présente à l'intérieur de la composition. Le terme se manifeste toujours sous la même forme, accolée et sans trait d'union. Aucune modification fonctionnelle n'a lieu, car le N2 détermine la catégorie du groupe nominal en anglais.

13 Le morphème composé, tel que freehold, semble pourtant apporter un élément de détermination de sens. Il est intéressant de noter que si l'on considère N2 d'une manière isolée, ce nom déverbal se trouve selon New General Service List (NGSL) ${ }^{11}$ parmi les mots les plus fréquents de la langue anglaise. En effet, hold se trouve à la $222^{\mathrm{e}}$ place 
des entrées les plus couramment utilisées et, de ce fait, il est polysémique dans la mesure où les variations de sens déterminent toutes sortes de possessions. Au sein du domaine du conveyancing, cependant, et considéré d'un point de vue diachronique, le nom semble avoir gardé un sens bien particulier. En effet, la construction du sens de " possession » de hold en tant que N2 provient du fait de tenir un terrain féodal au nom du souverain. Suivant la tradition anglo-saxonne, le nom déverbal est souvent employé dans l'expression have and hold, traduction du latin «habendum et tenendum », dont l'allitération renforce le pouvoir féodal sur le sol puisque « all the lands and tenements in England in the hands of subjects are holden mediately or immediately of the King " (Mellinkoff 1963 : 93). Une fois accolée à l'adjectif free, la composition change de sens, car l'adjectif réduit davantage le champ sémantique du nom déverbal. Désormais, le morphème composé précise un autre type de possession, celui d'une "propriété foncière perpétuelle et libre» (GDT). L'effet de sens de free dans cette structure correspond à un degré de détermination par rapport au type de possession, car il libère franchement la tenure de propriété de toute dépendance féodale. Cet effet est luimême infléchi par son appartenance à la structure déverbale, mais cet infléchissement correspond à une certaine restriction de sens dans la mesure où le champ sémantique de la composition de $\mathrm{N} 1$ et $\mathrm{N} 2$ vise plus la tenure d'une propriété foncière libre de toute dépendance hiérarchique. À ce stade, il est encore trop tôt pour conférer à cette composition l'éventuel statut d'un terme monosémique, car la forme du mot ne saurait garantir un seul sens unique.

Le deuxième type de morphème composé se caractérise par son aspect non accolé dont l'ordre d'arrangement séquentiel des mots est susceptible de produire des modifications au niveau du niveau du sens. Ces compositions concernent par exemple title abstract, title number, title plan, title report, title search, purchase title. Dans ces entités morphologiques, on voit que le mot title peut se trouver à différents endroits. Son sens, c'est-à-dire " ownership of real estate or personal property " (Nolo op. cit.) est-il pourtant mis à l'épreuve ? Il est vrai que l'anglais a tendance à aller du particulier au général, ce qui implique l'ordre déterminant-déterminé. Cet ordre coordonne les déterminants dans la mesure où le déterminé, au sens plus général, se rapporte au déterminant en le précisant ainsi. Employé dans le nom composé purchase title, le mot title acquiert le statut du déterminé tout en gardant sa dimension polysémique, car le déterminant précise juste le type de titre ; le déterminant distingue de cette manière le terme des autres types comme titre de créance ou titre de transport. Par conséquent, le champ sémantique ne se réduit que faiblement en renvoyant à un quelconque titre d'acquisition. Il ne fait d'ailleurs nulle part référence à un document unique et donc à un sens unique. Employé dans la composition title abstract, le mot title semble, en tant que déterminant, conférer au morphème composé une restriction de sens plus évidente. En effet, à chaque acte translatif ne correspond qu'un résumé de publication au Land Registry, et ce résumé est unique dès lors que la publication fait référence à ce title abstract. En théorie, title abstract est un titre spécifique tandis que dans le cas de purchase title plusieurs titres peuvent coexister. Or, l'élasticité sémantique de cette composition n'est guère réduite, car l'ordre séquentiel ne modifie que l'angle du déterminant. Dans le rôle du déterminant, le title devient une base lexicale importante et de cette manière, détermine le degré d'un angle nouveau en ce qui concerne le résumé de publication d'un acte translatif. Ce degré de détermination est d'ailleurs souvent lié au syntagme the abstract of the title dont le déterminant the singularise un élément de la notion du titre en question. Cependant l'effet de sens de la notion en elle- 
même n'est pas modifié, car cette nouvelle perspective éclaire simplement la notion historique du titre (" effet relatif »). L'arrangement séquentiel des sémantèmes en soi a donc peu d'effet sur la restriction de sens d'une telle composition, et en déduire une monosémie théorique serait une erreur. Les compositions telles que title abstract et purchase title, en revanche, constituent des bases morphologiques qui, dans une situation discursive très précise, adhèrent plus facilement au principe d'une monosémie potentielle. Nous y reviendrons ultérieurement.

Le dernier type de morphèmes nous permettant de montrer le possible lien entre forme et restriction de sens est la dérivation. Les morphèmes dérivés sont composés de flexions et d'affixes. Les flexions, comme le rappellent O. Ducrot et J.M. Schaeffer, " font partie de systèmes de conjugaison ou de déclinaison " (1972:432), tandis que les affixes " apparaissent avant ou après le sémantème " (ibidem). L'adjonction d'affixes à un sémantème, nommée dérivation, peut entraîner une modification de fonction. Par exemple, le mot conveyance est un nom déverbal puisque le suffixe -ance apporte une modification fonctionnelle au verbe convey. Le substantif renvoie au processus dénoté par le verbe, son sens originel polysémique semble persister : «transport or carry to a place» (OED). En revanche, le suffixe -ty réalise une dérivation à partir d'une base adjectivale telle que realty. Dérivé à partir de la base adjectivale simple real, ce mot se compose du suffixe invariable -ty, très courant en anglais, qui se greffe à la base lexicale. Cette postposition opère un changement de catégorie grammaticale faisant d'un adjectif un substantif. La postposition entraîne en outre une dérivation de sens, et plus précisément une extension de sens en ce qui concerne le domaine de la procédure translative de propriété. En effet, la suffixation provoque une extension vers la notion de bien immobilier tout en restant fidèle à sa base real, du fait que le terme realty désigne la "propriété franche d'un réel bien fond " (GDT $)^{12}$. Il est intéressant de noter que le suffixe -ty crée aussi une évaluation de condition, c'est-à-dire qu'il conditionne davantage la propriété physique à la réalité terrestre : « propriété comprenant le sol et tout ce qui en dépend en superficie et en profondeur » (GDT). En l'absence d'une telle condition, le terme realty serait privé de son réel sens. À la différence de conveyance, il serait dénudé de sens. Afin de vérifier la récurrence de ce phénomène, les exemples tels que property, certainty ou encore personality s'imposent, car, dans chacun de ces cas, le suffixe -ty est à l'origine d'une extension de sens vers le domaine du conveyancing.

À partir d'une base adjectivale, la dérivation donne naissance à un champ sémantique qui détermine soit un bien propre (proper-ty), la conviction certaine d'une proposition contractuelle (certain-ty), des droits liés à la capacité personnelle (physique ou juridique) contractuelle (personal-i-ty). Ces exemples montrent aussi que le sens originel de la base adjectivale semble persister dans le cadre de la procédure translative. Les affixes -ance et -ty apportent certainement un élément complémentaire dans la réduction de la visée sémantique d'un terme puisque la forme du dérivé produit un sens plus détaillé. La dérivation, tout comme la composition, peut alors se trouver à la source d'un sens plus restreint, mais en inférer une réelle monosémie serait prématuré.

$\mathrm{Du}$ fait de sa fonction formelle vis-à-vis d'une restriction de sens, la dérivation, comme la composition, permet la classification des termes tels que conveyance et realty parmi le vocabulaire spécialisé de l'anglais du droit. Ce classement devient possible lorsque la dérivation formelle produit une dérivation de sens qui permet de distinguer le morphème entre mot de la langue générale et terme technique. Cette distinction autorise une extraction quantitative puisque l'occurrence des termes spécialisés se 
laisse désormais chiffrer. Le tableau 2 présente une vue d'ensemble sur les entrées prises en compte et classées en fonction du type de contrat et de leur occurrence.

Tableau 2. Entrées prises en compte et classées en fonction du type de contrat et de leur occurrence

\begin{tabular}{|c|c|}
\hline $\begin{array}{l}\text { Type de } \\
\text { contrat }\end{array}$ & Entrées et occurrences \\
\hline $\begin{array}{l}\text { Residential } \\
\text { Freehold } \\
\text { Contract by } \\
\text { solicitor } \\
\text { (CSRFC) }\end{array}$ & $\begin{array}{l}\text { contract (51), property (36), buyer (35), seller (25), clause (23), conveyance (17), } \\
\text { delivery (17), completion (16), title number (15), transfer (12), annex (10), } \\
\text { mortgage (11), provision (9), epitome of title (5), purchase price (5), enter into } \\
\text { contract (4), freehold (4), parties (4), incorporating (4), schedule (4), agreement } \\
\text { (3), interpretation (3), remedy (3), personality (3), possession (3), covenant (2), } \\
\text { easement (2), exchange (2), formation (2), jurisdiction (2), pre-contract enquiries } \\
\text { (2), rate (2), agreed terms (1), root (1), encumbrances (1), power of attorney (1) }\end{array}$ \\
\hline $\begin{array}{l}\text { Standard } \\
\text { Conditions of } \\
\text { Sale by Law } \\
\text { Society } \\
\text { (SCSLS) }\end{array}$ & $\begin{array}{l}\text { buyer (91), seller (63), property (48), completion (37), leases (33), title number } \\
\text { (26), contract (25), conveyance (24), notice (21), deposit (19), party (19), parties } \\
\text { (10), transfer (19), contents (15), requisitions (13), exchange (7), purchase price } \\
\text { (7), occupation (7), apportionment (6), public requirement (6), mortgage (6), } \\
\text { physical state (6), accrued interest (5), rate (5), delivery (4), formation (4), } \\
\text { encumbrances (4), remedies (3), incorporating (2), third party (2), conveyancer } \\
\text { (2), have and hold the property (1), deed (1), freehold (1), root of title (1), formal } \\
\text { document (1), create legal rights (1) }\end{array}$ \\
\hline
\end{tabular}

Cet inventaire montre un relatif déséquilibre numérique dans la mesure où certains termes sont absents soit de l'un soit de l'autre contrat, ce qui peut déterminer le niveau professionnel d'un acte. L'absence d'un terme tel qu'agreement (SCSLS) dans l'un des contrats peut avoir des conséquences substantielles sur la formation et l'exécution contractuelle jusqu'à mettre en cause la validité du contrat même. Parmi les entrées les plus courantes se rangent les morphèmes composés et dérivés à tendance monosémique (conveyance, title number, mortgage, freehold). L'inventaire met aussi en évidence le nombre très élevé des autres unités lexicales, notamment les morphèmes simples à forte tendance polysémique (contract, property, buyer, seller). Par rapport à ces morphèmes simples, les morphèmes complexes sont plus rares, et leur rareté semble encore se confirmer si l'on prend en compte d'autres critères qualitatifs comme la référence et la notion du concept. C'est à ces critères que nous nous intéressons dans la section suivante.

\section{La monoréférentialité et l'uninotionnalité, sources de fragmentation de sens}

Lorsque l'on aborde la propriété qualitative d'un terme, des fondements à la fois linguistiques et terminologiques s'invitent dans le débat, car «les termes sont des unités qui représentent les objets de la réalité » (Cabré op.cit. : 80). En effet, le terme est une unité complexe qui se caractérise, sur le modèle du signe linguistique, par son aspect tridimensionnel réparti entre forme, sens et référent. Le triangle sémiotique 
schématise le fonctionnement du signe dans le langage, et pour fonctionner sémantiquement, le signe renvoie à un référent. Ce référent ne saurait guère être la réalité, comme un morphème tel que realty n'est jamais une maison réelle. C'est son référent qui émane plutôt de "l'imagination des individus " (Baylon \& Mignot op. cit. : 30), représentant ainsi une partie de la réalité. Le référent forme avec le signe cette unité linguistique, unité dont les terminologues s'inspirent pour définir, classer et établir des normes de la réalité du monde. La différence entre signe linguistique et unité terminologique est la primauté que donne la terminologie à la réalité sous forme d'objets matériels ou immatériels des divers domaines techniques et scientifiques. Ce sont ces objets qui prédominent dans le rapport entre signe et référent.

Ce rapport entre signe et référent comprend deux types de relation distincte. Les linguistes s'appuient sur la sémasiologie, c'est-à-dire les relations allant du signe aux objets du monde réel. Une telle relation prédispose l'existence de plusieurs référents par signe puisque le signe peut correspondre à plusieurs objets. Les terminologues en revanche préfèrent la démarche inverse, et plus précisément l'onomasiologie qui désigne les relations "allant des 'choses', des 'objets-à-nommer' aux signes » (Rey 1979 : 21). Dans ce cas de figure, un référent s'associe à un signe et la relation devient monoréférentielle à cause $\mathrm{du}$ rapport univoque entre référent et signe. La monoréférentialité, comme nous le verrons, a certainement une influence sur le sens du terme, mais il est trop tôt pour en déduire l'émergence d'une réelle monosémie, comme l'évoque Maria Teresa Cabré (1998: 82). Concernant l'exemple deed, une approche sémasiologique accorderait au signe deed plusieurs référents, c'est-à-dire plusieurs types de contrats. Les synonymes tels que legal document, contract, agreement, instrument en sont la preuve, ce qui confirme la polysémie du terme. Si l'on considère deed d'une manière onomasiologique, on part d'un objet précis du monde juridique pour établir sa référence avec le signe. Dans cette hypothèse, le terme ne saurait posséder qu'un référent, l'acte translatif de propriété, puisque le signe correspond à un seul référent. La référence devient monoréférentielle - un référent égale un signe - et univoque.

Or, ce qui complique encore le tableau, c'est que de nombreux signes se caractérisent par des relations onomasiologiques qui ne sont pas toujours univoques. En effet, le rapport entre référent et signe perd de son univocité lorsque le référent n'est pas clairement identifié ou lorsqu'il est multiple. Certes, le sens du terme deed dans une relation onomasiologique est plus ou moins précis dans la mesure où il s'inscrit dans le domaine du conveyancing: "a document that transfers ownership of real estate and is recorded in the local public land records " (Nolo op. cit.). Le référent, en l'occurrence l'acte translatif, ne garantit pourtant pas encore l'univocité du document ; il pourrait bien faire référence à deed of trust, deed in lieu of foreclosure ou encore transfert-on-death deed. La relation onomasiologique est loin de fixer une réelle monosémie du terme, car la monoréférentialité pourrait bien être pluriréférentielle en raison de la coexistence de plusieurs référents. Les relations dites onomasiologiques produisent néanmoins une fragmentation de sens, car à partir de chaque référent se crée un nouveau lien univoque avec le signe, et ainsi un nouveau sens. Cette fragmentation, qui fait coexister plusieurs référents par signe, a au moins deux effets sémantiques : le premier concerne la multiplication de sens, comme le montre l'exemple de deed qui peut bel et bien avoir comme référent spécifique "acte de fiducie ", "saisie-attribution" ou "dévolution successorale ». Chacun de ces référents est à l'origine d'une univocité qui fragmente 
davantage le sens global du terme. Le deuxième effet est davantage d'ordre qualitatif dans la mesure où il porte sur le degré de spécificité. En effet, un référent détaillé rend le sens d'un terme encore plus spécifique. Un acte de saisie-attribution, par exemple, est un contrat très spécifique qui désigne l'acquisition d'un bien par un créancier en cas de liquidation. Le référent détaille le sens dont la précision est basée sur l'idée d'un ensemble cohérent. Cette idée est coupée d'un ensemble conceptuel et porte, dans l'exemple de la saisie-attribution, sur l'extinction de la dette à l'égard du débiteur. Ce découpage est une fragmentation sémantique, car il scinde des fragments de sens issus d'un concept juridique, en l'occurrence l'acte translatif. La fragmentation découpe le sens d'un terme, mais en même temps, elle augmente aussi sa variation de sens. Si l'onomasiologie met en évidence cette fragmentation, elle ne saurait pour autant réduire le nombre de référents et les références qui en découlent. Le taux de variation de sens est encore élevé et la polysémie du terme est toujours présente. À ce stade, il n'est pas encore possible d'affirmer l'existence d'une monosémie théorique, même si le sens apparaît déjà très détaillé ${ }^{13}$.

Quant au rapprochement du principe de la référence et de l'émergence d'un potentiel monosémique, deux problématiques surgissent. L'une relève du cadre pragmatique, de l'énonciation, nous y reviendrons ultérieurement, l'autre concerne la dimension conceptuelle qu'entretient le référent. Le référent est ce à quoi renvoie un signe linguistique dans la réalité extralinguistique, d'où naît la relation référent-concept. Dès lors qu'on aborde le concept, on est amené à s'interroger sur des hypothèses empiriques puisqu'il sert aux individus à conceptualiser la réalité. De plus, le signe dont les individus se servent correspond à une représentation mentale qui s'organise sous forme de concept (Cabré op. cit. : 80-82). Si la représentation conceptuelle est une préoccupation des sémanticiens, la théorie générale de la terminologie s'appuie sur les relations notionnelles dans la dénomination d'un concept spécialisé, comme le détaille M. T. Cabré :

À la différence de la sémantique, qui s'intéresse à la relation entre la dénomination et le signifié, la terminologie se préoccupe d'abord de la relation qui s'établit entre l'objet réel et la notion qui le présente. C'est un processus d'abstraction que l'individu passe de cet objet réel à la notion ou à la catégorie d'objet. Pour ce faire, il fait abstraction des particularités de chacun des objets individuels et retient simplement celles qui sont pertinentes pour établir une catégorie regroupant ces objets avec leurs différences. (op. cit. : 31)

La notion représente les traits sémantiques pertinents d'un objet réel, E. Wüster parle d'ailleurs des "sciences des choses" pour identifier ce savoir-faire terminologique dans le but d'établir des catégories notionnelles. En effet, la notion est un découpage conceptuel du fait qu'elle découpe un seul trait significatif d'un ensemble conceptuel, ce qui est un avantage pour la pratique terminologique. Ainsi, les termes techniques peuvent être plus facilement identifiés, définis et classés.

Cependant, au niveau sémantique, la notion établit une relation uninotionnelle et, plus concrètement, la correspondance univoque entre notion et terme. De nouveau, on a affaire à une fragmentation de sens puisque le sens global du concept est morcelé en fractions notionnelles. Le terme freehold peut nous servir de modèle, car il incarne une notion spécifique à l'intérieur d'un concept global. Pour les professionnels du domaine, freehold signifie "a term for any interest in real estate" (Nolo op.cit.). Le terme appartient au vaste champ conceptuel du droit civil qui englobe le droit de propriété, d'usufruit, d'hypothèque immobilière ou de servitude. Si en revanche la notion 
pertinente de freehold est celle d'une tenure franche et perpétuelle, alors le terme développe une fragmentation de sens. Le terme devient conceptuellement solidaire dans un champ notionnel propre au domaine du droit des contrats. Le terme acquiert aussi une dimension uninotionnelle, car le rapport entre notion et terme est univoque. On peut ensuite plus facilement distinguer ce terme de son opposé sémantique leasehold qui désigne une "propriété à bail» (GDT). La notion pertinente de leasehold est celle d'une tenure conditionnelle limitée dans le temps. En principe, un bailleur peut facilement mettre un terme au droit de propriété de l'intéressé. Les termes tels que freehold et leasehold se désambiguïsent donc mutuellement à cause de leur uninotionnalité. L'uninotionnalité provoquerait-elle pour autant une monosémie potentielle? Le principe de l'uninotionnalité est à l'origine d'une certaine fragmentation de sens par le fait qu'il représente une fraction du concept global et qu'il établit une univocité assez étanche entre notion et terme. Cependant un terme comme freehold a-t-il vraiment un sens unique, plus précisément la tenue franche d'un bien dont on connaît exactement la géographie, l'effet relatif et les propriétaires? La réponse est clairement négative. Les acquisitions en freehold (ou en leasehold d'ailleurs) se comptent par milliers au sein d'une étude et aucune notion, par elle-même, ne pourra réellement affirmer la monosémie théorique du terme.

Les critères de la référence et de la notion témoignent de la relative variabilité de sens d'un même terme au sein d'un domaine spécialisé. La variabilité de sens se situe entre deux extrémités, la polysémie et la monosémie, et dépend des variations plus ou moins grandes selon les critères morphologiques, référentiels et notionnels. Si l'on considère ces critères d'une manière isolée, la tendance à la monosémie est faible puisque la restriction de sens est insuffisante. Le potentiel monosémique devient plus fort lorsque les critères morphologiques, référentiels et notionnels entrent en dialogue, car ils réduisent, fragmentent et découpent davantage le sens d'un terme. Le dialogue réduit les variations possibles, ce qui peut résulter en une monosémie théorique. Une analyse portant sur une telle monosémie réclame alors un raisonnement en matière de « gradient de potentiel ». Par gradient de potentiel, nous entendons plusieurs degrés de monosémie qui varient d'un potentiel faible jusqu'à un potentiel fort. Le potentiel faible concerne une restriction de sens insuffisante pour affirmer une monosémie, car le terme ne voit son élasticité sémantique que légèrement réduite. Dans ce cas, la dimension polysémique du terme demeure présente. Le potentiel fort, en revanche, détermine une restriction de sens qui autorise très peu de variations sémantiques. Plus la restriction est grande, plus on s'approche d'une monosémie théorique, et plus élevé est le degré d'un sens unique.

Pour la langue de spécialité, le raisonnement en termes de gradient de potentiel monosémique a une fonction particulière : il facilite le meilleur classement des termes en domaines conceptuels. Ce classement produit une hiérarchisation des entrées fondées sur le haut degré de spécialisation sémantique d'un terme. Cette spécialisation est possible une fois que la notion et la référence d'un terme sont sans équivoque et que la variation sémantique est réduite au plus haut degré. Les occurrences figurant dans le tableau 3 illustrent un tel classement; ce dernier est d'ailleurs très courant dans la pratique juridique puisqu'il s'agit des procédures translatives relatives à la formation, à l'exécution et aux effets d'un acte. 
Tableau 3. Entrées prises en compte et classées en fonction des domaines d'application

\begin{tabular}{|l|l|}
\hline $\begin{array}{l}\text { Domaines et sous- } \\
\text { domaines }\end{array}$ & Entrées prises en compte \\
\hline 1/ Formation & Agreement, intention, consideration, estoppel \\
\hline $\begin{array}{l}\text { Ex. sous-domaine } \\
\text { «consideration » }\end{array}$ & $\begin{array}{l}\text { Executory, exchange, purchase price, good reason, certainty, parole } \\
\text { evidence, conduct, performance }\end{array}$ \\
\hline 2/ Exécution & $\begin{array}{l}\text { Deed, incorporation, evidenced in writing, signature, capactiy, } \\
\text { delivery, completion }\end{array}$ \\
\hline Ex. sous-domaine «deed» & Formalism, in writing, express terms, material terms \\
\hline 3/ Effets & Property, title, binding, land registry \\
\hline Ex. sous-domaine « realty & $\begin{array}{l}\text { Real property, personal property, tangible property, freehold, } \\
\text { leasehold }\end{array}$ \\
\hline
\end{tabular}

Le corps gras de la colonne de gauche dans le tableau 3 signale les domaines clés dans le droit des contrats qui sont connus, compris et partagés par les juristes du domaine. Dans les cellules en dessous de chaque domaine clé se trouve l'exemplier d'un sousdomaine dont les termes ont fait l'objet d'une étude approfondie dans notre travail. À chaque catégorie de domaine dans la colonne de gauche correspondent à droite les entrées réservées aux discours des spécialistes (agreement, intention, consideration, estoppel, etc.). Ainsi, le terme consideration forme le sous-domaine de la « contrevaleur » appartenant au domaine plus large de la formation d'un contrat. Les termes tels que executory, exchange, good reason, etc. forment quant à eux un nouveau sous-domaine directement lié au domaine du droit mutuel de consideration.

L'appartenance des termes à des domaines structurés en système de classement témoigne des savoirs spécialisés, mais, et c'est le plus essentiel, elle se fonde sur la restriction du sens. Chaque domaine de spécialité possède de tels systèmes que l'on appelle en terminologie un "arbre de domaine ». Cet arbre schématise l'appartenance structurée des termes à un ensemble cohérent ; l'arbre reflète également les frontières sémantiques des termes parce que chaque terme est classé en fonction de sa pertinence de sens inhérente au domaine spécifique. Au-delà du classement quantitatif en domaine, le raisonnement en matière de gradient permet la structuration arborescente des termes puisque l'univocité entre la forme et le référent d'un côté et la forme et la notion de l'autre restreignent le taux de variation de sens. La polysémie interne semble d'ailleurs perdre du terrain vis-à-vis d'une monosémie potentielle puisque la structure arborescente ne peut fonctionner en présence d'une délimitation du champ sémantique des termes et des domaines. Le gradient de potentiel monosémique représente de cette manière une ouverture au contenu empirique des concepts contractuels, car il fait apparaître les contours précis d'un champ sémantique restreint. Ces contours sont le fondement même d'une émergence du sens unique d'un terme et de la structuration arborescente qui en découle. 
Les paragraphes qui précèdent ont mis l'accent sur un potentiel monosémique théorique. Or, le degré de sens unique est plus élevé lorsqu'il s'agit de considérer l'emploi monosémique des termes. C'est la raison pour laquelle, dans la dernière section, nous procédons à la recontextualisation des termes dans le cadre de la communication spécialisée.

\section{La communication spécialisée, un critère essentiel pour affirmer la monosémie}

Selon Ludwig Wittgenstein, «le sens d'un mot, c'est son emploi dans la langue $»^{14}$ (1953 : 311), ce qui nous amène à nous interroger sur la nécessité d'intégrer à l'étude du potentiel monosémique des indications communicatives liées à l'emploi des termes. Les termes, comme nous l'avons montré, sont tant des unités de la langue que des éléments de connaissance. Ils sont également "des véhicules de communication » (Cabré op. cit. : 61). En effet, le premier objectif d'un terme est son emploi dans les communications spécialisées, ce qui "permet aux spécialistes d'échanger des connaissances sur un domaine " (op. cit. : 90). Selon T. Cabré, la communication spécialisée se distingue de la communication générale par plusieurs aspects. D'abord, les interlocuteurs, c'est-à-dire l'émetteur et le récepteur, « sont plus ou moins spécialistes d'un domaine, et ils savent d'entrée de jeu qu'ils partagent certaines informations sur le domaine en question » (op. cit. : 92). C'est notamment le cas avec le terme freehold: un solicitor anglais applique les informations référentielles et notionnelles lorsqu'il s'agit de rédiger un acte translatif. Avant toute rédaction même, il doit faire la distinction par rapport à une acquisition en freehold ou en leasehold puisque la trame rédactionnelle de l'acte en dépend. En dehors de la pratique du conveyancing, ces informations perdent leur signification, ce qui confirme non seulement l'efficacité de la communication intraprofessionnelle, mais aussi l'invariabilité du terme. Les termes tels que freehold et leasehold deviennent-ils alors monosémiques dans le cadre d'une communication spécialisée? Pour répondre à cette question, il conviendrait d'aborder un dernier élément lié à la communication spécialisée.

31 L'échange des connaissances dans un domaine spécialisé se fait le plus souvent par l'intermédiaire d'un texte qui, d'après T. Cabré, « est fondamentalement informatif et descriptif» (Cabré op. cit.: 92). À l'exemple des actes translatifs, ces textes sont caractérisés « par la concision, la précision et l'adéquation » (ibidem). Le terme purchase price mérite d'être examiné ici, car il illustre la manière dont un texte spécialisé peut éliminer toute variation de sens possible. La notion de purchase price indique un «prix qui est payé en contrepartie d'un bien ou d'un service » (GDT). La contrepartie n'a pas forcément besoin de faire référence à la valeur réelle du bien, car il s'agit ici d'un concept de la common law par laquelle les parties contractantes s'accordent sur un droit mutuel. Le droit mutuel en revanche correspond à une valeur financière précise : "The Seller will sell and the Buyer will buy the Property, for the Purchase Price on the terms of the contract» (CSRFC $\S 2.1$ ). Cette valeur ne laisse aucune marge d'erreur, car elle correspond au prix contracté. Le caractère mutuel du prix révèle ainsi deux aspects : le premier est l'invariant de la contrepartie contractée ; le deuxième aspect concerne le contexte d'emploi très précis qui n'autorise qu'un prix d'achat par acte authentique. Les conditions d'emploi du terme semblent donc produire une restriction de sens conséquente. Plus l'on réduit le contexte d'emploi, plus on peut supposer une tendance 
vers un emploi monosémique du terme. En effet, toute forme d'ambiguïté est éliminée dans un tel énoncé, car toute variation de prix est exclue. Par conséquent, le taux de variation de sens est extrêmement minime et un emploi monosémique peut s'affirmer.

À présent, il importe de déterminer la manière dont on peut vérifier un emploi monosémique. L'un des moyens de vérification est le principe de l'univocité, et plus précisément de la biunivocité. La biunivocité s'inspire des concepts logiques et mathématiques qui font du principe un argument en faveur d'un élément qui entraîne toujours le même corrélatif. La biunivocité et la réciprocité sont complémentaires, complémentarité qui permet le repérage biunivoque d'un trait sémantique. Selon $\mathrm{E}$. Wüster, « on doit exiger de la terminologie, pour emprunter une expression empruntée aux mathématiques, biunivoque, c'est-à-dire que, fondamentalement, à chaque concept n'est attribué qu'une seule signification, et inversement" (Wüster op. cit.: 79) ${ }^{15}$. Ce principe (Ein-eindeutigkeit) peut être critiqué pour sa rigidité, mais il représente néanmoins un atout pour vérifier la monosémie d'un terme dans le cadre de son énonciation. La relation réciproque confère au terme un rapport rigide entre forme et dénomination écartant toute plasticité sémantique. Le terme résiste de cette manière aux efforts de multiplication de sens dévoilant une clarté entre signe et notion, entre forme et dénomination. Cette clarté (E. Wüster évoque l'adjectif eindeutig) désigne à la fois une exclusivité de sens ainsi qu'une évidence corrélative. Le rapport devient sûr et évident, le sens du terme unique et identifiable. Le taux de variation de sens est désormais infiniment bas puisque les autres variants de sens ont été écartés. Le gradient de potentiel monosémique atteint son niveau le plus élevé en faisant émerger un seul sens clair et net par terme. L'emploi monosémique d'un terme est alors attesté. Prenons une nouvelle fois l'exemple de purchase price dont la monosémie semble s'affirmer si la biunivocité du terme peut être vérifiée par un numéro unique de référence de publication. Ce numéro permet en effet l'identification réciproque entre acte et prix. Dans ce cas, et seulement dans ce cas, le numéro d'enregistrement permet l'identification du seul et réel prix d'achat stipulé dans l'acte et, inversement, la valeur financière exacte n'a de sens qu'au sein de cette transaction précise. Très souvent dans la pratique du conveyancing, la présence d'un déterminant, en l'occurrence l'article défini, apporte une détermination syntaxique supplémentaire au terme en le quantifiant comme unique: "The amount payable by the buyer on completion is the purchase price» (SCSLS $\& 6.4)^{16}$. Le déterminant ne nécessite aucune préposition complémentaire, car le prix d'acquisition est la seule contrevaleur de l'acte. Le terme faisant référence au seul droit mutuel élimine toute ambiguïté, car la contrepartie est un élément contractuel avéré. Ainsi, le terme est ein-eindeutig, sa reconnaissance formelle et incontestable et son emploi monosémique sont acquis. Il est intéressant de noter que la biunivocité du prix d'achat n'est pas propre au seul domaine du conveyancing, mais à tout contrat. En droit civil, toutes sortes de contrats, de l'acquisition d'un titre de transport jusqu'à la cession des parts sociales d'une société civile, s'appuient effectivement sur cet aspect biunivoque du prix. Ainsi, la biunivocité entre forme et dénomination dévoile un paramètre sémantico-terminologique que l'on retrouve au-delà du caractère particulièrement technique de la procédure translative de propriété. 


\section{Conclusion}

En repensant les sources de la monosémie et en s'appuyant sur le gradient de potentiel, l'étude sur la monosémie des termes ouvre de nouveaux espaces de réflexion. Ces réflexions éclairent davantage la différence entre monosémie théorique et emploi monosémique des termes. L'identification de critères définitoires propres au domaine du conveyancing pourrait également fournir un élément majeur de la tendance à la monosémie pour d'autres domaines. Cette étude n'a pas pu prendre en compte une perspective contrastive, mais il serait sans doute intéressant de considérer la traduction des termes monosémiques. Enfin, il serait aussi passionnant de savoir si la monosémie des termes juridiques émerge dans d'autres aires géographiques, dans d'autres juridictions du monde anglophone.

\section{BIBLIOGRAPHIE}

BAYLON, Christian \& Xavier MIGNOT. 2000. Initiation à la sémantique du langage. Paris : Nathan. BRÉAL, Michel. 1897. Essai de sémantique (science des significations). Paris : Hachette.

CABRÉ, Maria Teresa. 1998. La Terminologie, théories, méthodes et applications. Ottawa : Les Presses de l'Université d'Ottawa.

CONDAMINES, Anne. 2005. Sémantique et corpus. Paris : Hermes-Science.

CRUSE, Alan. 2004. Meaning in Language: An Introduction to Semantics and Pragmatics. Oxford : Oxford University Press.

DELAVIGNE, Valérie \& Myriam BOUVERET. 2000. Sémantique des termes spécialisés. Rouen : Publications de l'Université de Rouen - C.N.R.S.

DEPECKER, Loïc. 2002. Entre signe et concept. Éléments de terminologie générale. Paris : Presses Sorbonne Nouvelle.

DUCROT, Oswald \& Jean-Marie SCHAEFFER. 1972. Dictionnaire encyclopédique des sciences du langage. Paris : Seuil.

DUXBURY, Robert. 2008. Contract Law. Londres : Sweet \& Maxwell.

FERRARI, Laura. 2002. « Un caso de polisemia en el discurso juridico ?». Terminology 8/2, 221-222.

GILBERT, Louis. 1973. «La spécificité du terme scientifique et technique ». Langue française 17/1, 517.

GLEDHILL, Chris \& Natalie KÜBLER. 2016. « What can linguistic approaches bring to English for specific purposes? ». ASp 69, 65-95.

LARRIVÉE, Pierre. 2008. Une histoire du sens. Bruxelles : Peter Lang.

LAW, Jonathan. 2015. A Dictionary of Law. Oxford : Oxford University Press. 
Le Grand Dictionnaire Terminologique. (GDT). [en ligne] Office québécois de la langue française. < http://www.granddictionnaire.com>. Consulté le 15 novembre 2016.

LERAT, Pierre. 1997. « Approches linguistiques des langues spécialisées ». ASp 15-18, 1-10.

LESCOT, Adeline. 2013. Bescherelle Le vocabulaire pour tous. Paris : Hatier.

MELLINKOFF, David. 1963. The Language of Law. Eugene, OR : Wipf and Stock.

MILNER, Jean-Claude. 1989. Introduction à une science du langage. Paris : Seuil.

Nolo's Free Dictionnary of Law Terms and Legal Definitions (Nolo). [en ligne] Berkeley, CA 94710.

<http://www.nolo.com/dictionary>. Consulté le 2 janvier 2017.

NORTHCOTt, Jill. 2012. « Legal English ». In PALTRIDGE, B. \& S. STARFIED (dir.), Handbook of English for Specific Purposes. Wiley-Blackwell : Londres, 213-226.

PALTRIDGE, Brian \& Sue STARFIELD. 2012. Handbook of English for Specific Purposes. Londres : WileyBlackwell.

PEASON, Jennifer. 1998. Terms in Context. Amsterdam \& Philadelphie : John Benjamins.

RESCHE, Catherine. 2013. Economic Terms and Beyond: Capitalising on the Wealth of Notions. Collection Linguistic Insights $n^{\circ} 176$. Berne : Peter Lang.

REY, Alain. 1979. La terminologie : noms et notions. Paris : Presses Universitaires de France.

SAGER, Juan C. 2000. Essays on Definition. Amsterdam : J. Benjamins.

SAUSSURE, Ferdinand de. 1995 [1916]. Cours de linguistique générale. Paris : Payot.

TAMBA, Irène. 2007. La sémantique. Paris : Presses Universitaires de France.

THOIRON, Philippe \& Henri BÉJOINT. 2010. « La terminologie, une question de termes? ». Meta :

journal des traducteurs, 55/1, 105-118.

TEMMERMAN, Rita. 2000. Towards New Ways of Terminology Description. The Sociocognitive

Approach. Amsterdam : John Benjamins.

TIERSMA, Peter. 1999. Legal Language. Chicago: The University of Chicago Press.

WILLIAMS, Geoffrey. 2003. « From meaning to words and back: Corpus linguistics and specialised lexicography ». ASp 39-40, 91-106.

WITTGENSTEIN, Ludwig. 1953. Philosophische Untersuchungen. Stuttgart : Macmillan.

WÜSTER, Eugen. 1979. Einführung in die allgemeine Terminologielehre und terminologische Lexikographie. 3. Auflage. Bonn : Romantischer Verlag.

\section{NOTES}

1. La common law est un système juridique issu du droit anglais qui est fondé sur le principe casuistique dont la principale source est la règle du précédent. Elle est implantée en Angleterre et au Pays de Galles ainsi que dans la majorité des anciennes colonies britanniques, tandis que l'Écosse se caractérise par un système juridique mixte entre droit civiliste et common law (voir Duxbury [2008]).

2. Dans notre étude, le signe est considéré en termes linguistiques et terminologiques dont nous préciserons les significations dans la section 1. 
3. L'acronyme «SCSLS » se décline en Standard Conditions of Sale, the Law Society, celui de " CSRFC» en Contract for the sale of Residential Freehold Property. La Law Society of England and Wales est une association professionnelle qui représente la profession juridique au sein de la juridiction de l'Angleterre et du Pays de Galles.

4. Notre traduction.

5. «Die Sprache besteht jedoch überwiegend aus Wörtern, die [...] als Benennungen unmittelbar den Begriffen zugeordnet sind » (Wüster 1979: 6). Notre traduction.

6. < http://cnrtl.fr/definition/monosémie>. Consulté le 15 avril 2017.

7. Une histoire de la sémantique linguistique se trouve dans Larrivée (2008).

8. Une clarification semble s'imposer ici : elle porte sur la différence entre les termes mot et nom. Le mot s'analyse au sens saussurien à l'intérieur du système langagier, tandis que le nom est plutôt utilisé par les terminologues. En effet, Rey précise que «le nom renvoie à un élément distinct de l'expérience humaine [...] qu'il désigne ou dénote » (Rey 1979 : 21).

9. Dans le cadre restrictif de cette étude, nous avons choisi de ne pas développer la morphologie des syntagmes et leur importance vis-à-vis de la restriction de sens des termes. Il convient néanmoins de noter que les entrées comme to have and to hold et estoppel by deed réalisent, du fait de leur forme spécifique, une restriction de sens dans la mesure où leur sens peut sous certaines conditions dévoiler un potentiel monosémique.

10. Les mots simples présentent peu d'intérêt dans la considération du rapport entre morphologie et restriction de sens. Ils s'avèrent plus intéressants lorsqu'il s'agit d'analyser la référence et la notion du concept. Nous y reviendrons ultérieurement.

11. Cette liste s'appuie sur les mots du Cambridge English Corpus et couvre plus de $92 \%$ des mots les plus fréquents dans les textes anglais (http://www.newgeneralservicelist.org>, consulté le 15 avril 2017).

12. Il s'agit de nos italiques.

13. Dans le but de réduire davantage la variation du sens d'un terme, on pourrait envisager l'importance de la référence actuelle. Élaborée par le linguiste Jean-Claude Milner (1989), la référence virtuelle est d'ordre général tandis que la référence actuelle concerne l'évocation effective de référent. Même si la référence actuelle contient un référent spécifique au sein d'une énonciation (voir section 4), elle ne pourra garantir une monosémie potentielle, car les liens entre signe et référent peuvent être multiples.

14. "Die Bedeutung eines Wortes ist sein Gebrauch in der Sprache» (Wittgenstein $1953: 311$ ). Notre traduction.

15. "[Es ist] in der Terminologie zu verlangen, dass sie, um einen Ausdruck aus der Mathematik zu gebrauchen, eineindeutig ist. D.h., dass grundsätzlich jedem Begriff nur eine einzige Benennung zugeordnet ist, und umgekehrt » (Wüster 1979 : 79). Notre traduction.

16. Il s'agit de nos italiques.

\section{RÉSUMÉS}

Cette étude vise à analyser le potentiel de la monosémie concernant les termes de l'anglais du droit appartenant au domaine du droit des contrats, et plus précisément le conveyancing, la procédure translative de propriété. La monosémie, un caractère sémantique qui affirme un seul sens spécifique, est un sujet assez peu étudié par la recherche contemporaine. Or, la monosémie 
semble conférer au terme son degré de spécificité en réduisant la plasticité sémantique inhérente à la polysémie. Après un bref rappel des questions théoriques, les critères morpho-lexicaux, référentiels, conceptuels et communicatifs sont analysés pour déterminer les sources d'une éventuelle monosémie. Une quarantaine d'entrées extraites d'un corpus juridique est présentée à travers une approche sémantico-terminologique afin de faire émerger un gradient de potentiel qui s'avère pertinent pour la monosémie théorique ainsi que l'emploi monosémique des termes, mais également pour mettre en évidence la spécificité sémantique du vocabulaire spécialisé de l'anglais du droit.

The purpose of this article is to look at a possible monosemy of legal terms taken from English conveyancing practices, the legal process of transferring property rights from one owner to another. Monosemy, the univocal relationship between a term and its meaning, appears to be a phenomenon less frequently studied. Yet, the possession of one meaning appears to be an inherent property of terms from specialised languages in specific contexts. After establishing a theoretical framework and reviewing previous research, the article analyses the emergence of sense boundaries through morpholexical, referential, conceptual and communicative criteria. The recognition for a gradient potential in sense boundaries appears to become necessary when it comes to shedding light upon the difference between a theoretical and pragmatic monosemy of English conveyancing terms, a gradient potential intrinsically related to English for Specific Purposes in that it helps clarify, stabilise and confirm the semantics of technical terms.

\section{INDEX}

Mots-clés : anglais du droit, communication spécialisée, droit des contrats, monosémie, morphologie, polysémie, restriction de sens, terminologie

Keywords : contract law, legal English, monosemy, morphology, polysemy, sense boundaries, specialised communication, terminology

\section{AUTEUR}

\section{ANDREAS PICHLER}

Andreas Pichler a un double parcours juriste et angliciste. Formé initialement comme juriste en droit privé international, il a été admis au concours de clerc de notaire et a travaillé dans des cabinets d'avocats dans le monde anglophone et dans des études notariales. Il a été qualifié aux fonctions de maître de conférences en 2016. Après avoir travaillé à Aix-Marseille Université pendant une dizaine d'années, il a quitté l'enseignement supérieur en 2017 afin d'intégrer une étude des Notaires de Paris en tant que notaire assistant. pichler.pro@gmail.com 